\title{
Nuclear Microprobe Studies of the Electronic Transport Properties of Cadmium Zinc Telluride (CZT) Radiation Detectors ${ }^{\text {th }}$
}

\author{
G.Vizkelethy ${ }^{*, a, b}$, B.L.Doyle ${ }^{\mathrm{b}}$, D.S.Walsh ${ }^{\mathrm{b}}$, R.B.James ${ }^{\mathrm{c}}$ \\ ${ }^{a}$ Idaho State University, Pocatello, ID, 83209 \\ ${ }^{\mathrm{b}}$ Sandia National Laboratories, Albuquerque, NM \\ ${ }^{\mathrm{c}}$ Sandia National Laboratories, Livermore, CA
}

\begin{abstract}
Ion Beam Induced Charge Collection (IBICC) is a proven albeit relatively new method to measure the electronic transport properties of room temperature radiation detectors. Using an ion microbeam, the charge collection efficiency of CZT detectors can be mapped with submicron resolution and maps of the electron mobility and lifetime can be calculated. The nuclear microprobe can be used not only for characterizing detectors but also with the use of Time Resolved IBICC (TRIBICC) and lateral IBICC/TRIBICC we can deduce information about the electron and hole mobility and lifetime profiles, and about the variation of electric field along the detectors' axes. The Sandia Nuclear Microprobe has been and is being used routinely to characterize CZT detectors and measure their electronic transport properties. In this paper we will present the results of these measurements for different detectors. Furthermore the damage effects caused by the probing beam will be discussed and a simple model will be presented to explain the characteristic charge collection efficiency pattern observed after high dose irradiation.
\end{abstract}

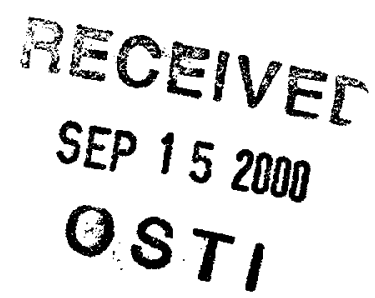

Keywords: IBICC, TRIBICC, nuclear microprobe, CZT, electronic transport properties

\section{INTRODUCTION}

Room temperature radiation detectors are usually tested using alpha particle sources ${ }^{\mathrm{I}}$. The resolution of these measurements is in the order of a few hundred $\mu \mathrm{m}$ since apertures are used to focus the alpha particle beam. In many cases a better resolution than that is preferred since features on these detectors have much smaller characteristic sizes than hundred $\mu \mathrm{m}$. Nuclear microprobes, which are usually used for testing the radiation hardness of microelectronic circuits or measure composition of various samples on the microscopic scale, are the perfect tools to study the response of radiation detectors on microscopic scale. The techniques known as Ion $\underline{B}$ eam Induced $\underline{\text { Charge }}$ Collection (IBICC) and Time Resolved IBICC (TRIBICC) in the microprobe community allow one to determine the charge collection efficiency, electron and hole mobilities and lifetimes, and the electric field along the detectors axis with micrometer resolution. There are two versions of the above two techniques, frontal and lateral. We call the technique frontal when one of the detector's electrodes is irradiated. This allows us to determine the charge collection efficiency and an average $\mu \tau$ product from the IBICC measurement for the electrons or holes. When TRIBICC is used, both the mobility $(\mu)$ and the lifetime $(\tau)$ can independently be determined. In case of lateral (TR)IBICC, when the side of the detector is irradiated, information can be deduced about the electric field along the detector's axis. In this paper we will give a brief overview of these techniques illustrated with examples on two different detectors.

\section{EXPERIMENTAL}

These experiments were carried out using the Sandia microbeam facility which is described in detail elsewhere ${ }^{2,3}$. Here we will restrict ourselves to a very brief description needed to understand the procedure. Figure 1 shows the schematics of the experimental setup. The high-energy (several $\mathrm{MeV}$ ) ion beam from a tandem particle accelerator is focused typically to a one- $\mu \mathrm{m}$ spot using a magnetic lens. Then the ion beam was scanned over a larger area - typically about $200 \times 180 \mu \mathrm{m}^{2}-$ using electrostatic scanning. When the side of the detector was scanned in its whole length the sample was moved mechanically at about $1 \mu \mathrm{m}$ precision after each scan to the next area. The individual scans were merged into a larger map off-line. In an IBICC experiment the signal from the detector was processed with a standard charge sensitive preamplifier and with a spectroscopy amplifier. The measured signal (using a proper shaping time) is proportional to the total collected charge, $Q$. The

\footnotetext{
${ }^{\uparrow}$ Sandia is a multiprogram laboratory operated by Sandia Corporation, a Lockheed Martin Company, for the United States Department of Energy under Contract DE-AC04-94AL85000.

- Correspondence: Email: gvizkel@sandia.gov, Telephone: (505) 284-3120, Fax: (505) 844-7775
} 


\section{DISCLAIMER}

This report was prepared as an account of work sponsored by an agency of the United States Government. Neither the United States Government nor any agency thereof, nor any of their employees, make any warranty, express or implied, or assumes any legal liability or responsibility for the accuracy, completeness, or usefulness of any information, apparatus, product, or process disclosed, or represents that its use would not infringe privately owned rights. Reference herein to any specific commercial product, process, or service by trade name, trademark, manufacturer, or otherwise does not necessarily constitute or imply its endorsement, recommendation, or favoring by the United States Government or any agency thereof. The views and opinions of authors expressed herein do not necessarily state or reflect those of the United States Government or any agency thereof. 


\section{DISCLAIMER}

Portions of this document may be illegible in electronic image products. Images are produced from the best available original document. 
signals were recorded in $512 \times 512$ maps and the data was later compressed to $64 \times 64$ maps to improve statistics. The typical particle current in these experiments was about $100 \mathrm{cps}$ in order to avoid damage to the scanned spot.

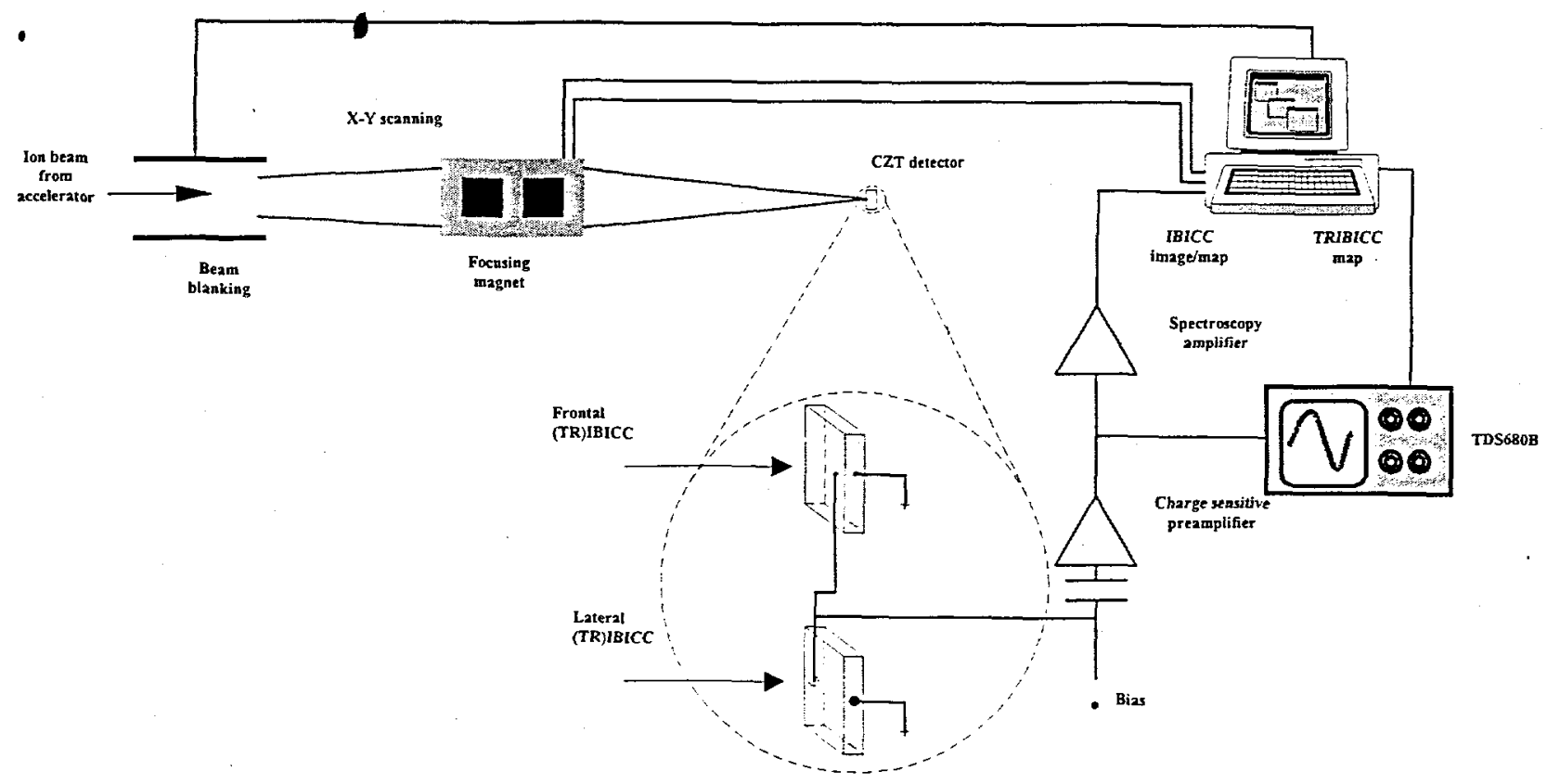

Figure 1 Experimental setup

While the IBICC signal measures $Q$, the TRIBICC measurement gives both $Q$ and the drift time, $T_{r}$. In the case of the TRIBICC experiments the preamplifier signal was digitized and recorded using a fast, computer controlled Tektronix TDS 680B oscilloscope ( $1 \mathrm{GHz}$ analog equivalent). Beam damage effects and multiple ion-strikes were eliminated using a high speed ( $<25$ ns rise time) on demand beam deflector system (shown in Figure 1) to remove the ion beam from the detector during the relatively long signal processing time (it typically took about $100-200 \mathrm{~ms}$ to digitize the signal at 500 to 1000 point resolution, transfer and store in the computer). This way each recorded transient was the result of a single ion strike at that particular point in the scan.

We used two different CZT detectors in these experiments. Both were produced using a high pressure Bridgman process. One was a spectroscopy grade, $10 \mathrm{~mm} \times 10 \mathrm{~mm} \times 2 \mathrm{~mm}$, single crystal detector with sputtered platinum contacts (Detector 1). The other detector was a passivated, doped one with gold contacts made by electrochemical deposition (Detector 2). The size of this detector was $8 \mathrm{~mm} \times 10 \mathrm{~mm} \times 2 \mathrm{~mm}$. We usually use a $5.4 \mathrm{MeV}$ He beam in our experiments to be able to compare our result to the ones obtained using radioactive alpha sources. In order to show the effect of the type of the probing beam we carried out experiments using a $4.5 \mathrm{MeV}$ proton beam on the second detector.

The charge collection efficiencies were calculated by normalizing both the IBICC and TRIBICC signals to responses from a Si PIN diode taking into account the different electron-hole pair creation energies $\left(\varepsilon_{e-h}^{S i}=3.6 \mathrm{eV}\right.$ and $\varepsilon_{e-h}^{C Z T}=4.67 \mathrm{eV}$ were used).

\section{RESULTS AND DISCUSSION}

\subsection{Frontal IBICC and TRIBICC}

The induced charge on the contacts of a planar detector is given by the Hecht equation ${ }^{45}$,

$$
Q(x)=N_{0} e\left[\frac{\lambda_{e}}{d}\left(1-e^{-\frac{x_{e}}{\lambda_{e}}}\right)+\frac{\lambda_{h}}{d}\left(1-e^{-\frac{x_{h}}{\lambda_{h}}}\right)\right]
$$


where $N_{0}$ is the number of electron-hole pairs created, $e$ is the unit electric charge, $d$ is the thickness of the detector, $\lambda_{e}$ and $\lambda_{h}$

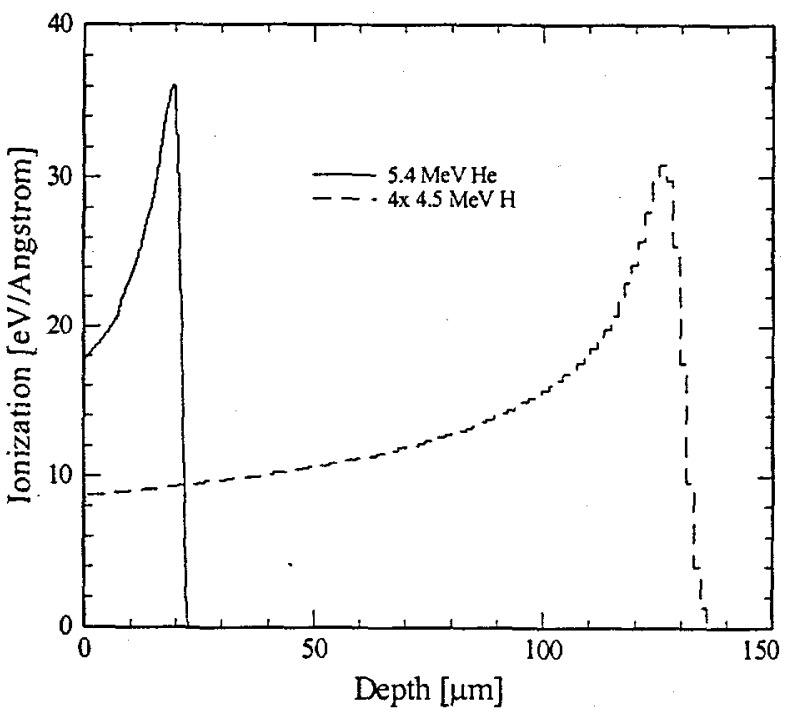

Figure 2 Ionization profiles of $5.4 \mathrm{MeV}$ alpha particles and $4.5 \mathrm{MeV}$ protons are the electron and hole drift lengths, and $x_{e}$ and $x_{h}$ are the distances the electrons and holes have to travel to reach their collecting electrodes. Using a few $\mathrm{MeV}$ alpha particles one of the charge carriers can be eliminated from (3.1) since all the charge is created in a few $\mu \mathrm{m}$ region below the irradiated electrode. Figure 2 shows the ionization profile of a $5.4 \mathrm{MeV}$ alpha particle calculated by the SRIM code. From the figure we can see that all the charge is generated in the top $20 \mu \mathrm{m}$ layer. This distance is much smaller that the usual thickness of this type of detectors; therefore, (3.1) can be simplified to the single carrier Hecht equation for the case when the cathode is irradiated,

$$
Q=N_{0} e \frac{\lambda_{e}}{d}\left(1-e^{-\frac{d}{\lambda e}}\right)
$$

since $d-x \approx d$. Since the drift length is given by $\lambda=\mu \cdot \tau \cdot E$ with $\mu$ being the mobility, $\tau$ the lifetime, and $E$ the applied electric field, we can determine the $\mu \tau$ product by measuring the charge collection efficiency at different bias voltages (IBICC measurement). In many cases this is sufficient since the $\mu \tau$ product mainly determines the quality of the detector. When the mobility or lifetime is required the signal transient should be recorded (TRIBICC) and from the signal rise time an average drift velocity can be calculated. These signal transients were measured for both detectors in the range of $50 \mathrm{~V}$ to $200 \mathrm{~V}$ for detector 1 and from 50 to $400 \mathrm{~V}$ for detector 2. Detector 1 had high leakage current even at $200 \mathrm{~V}$ bias $(-0.5 \mu \mathrm{A})$, therefore, we could not apply higher voltages. From the transients the rise
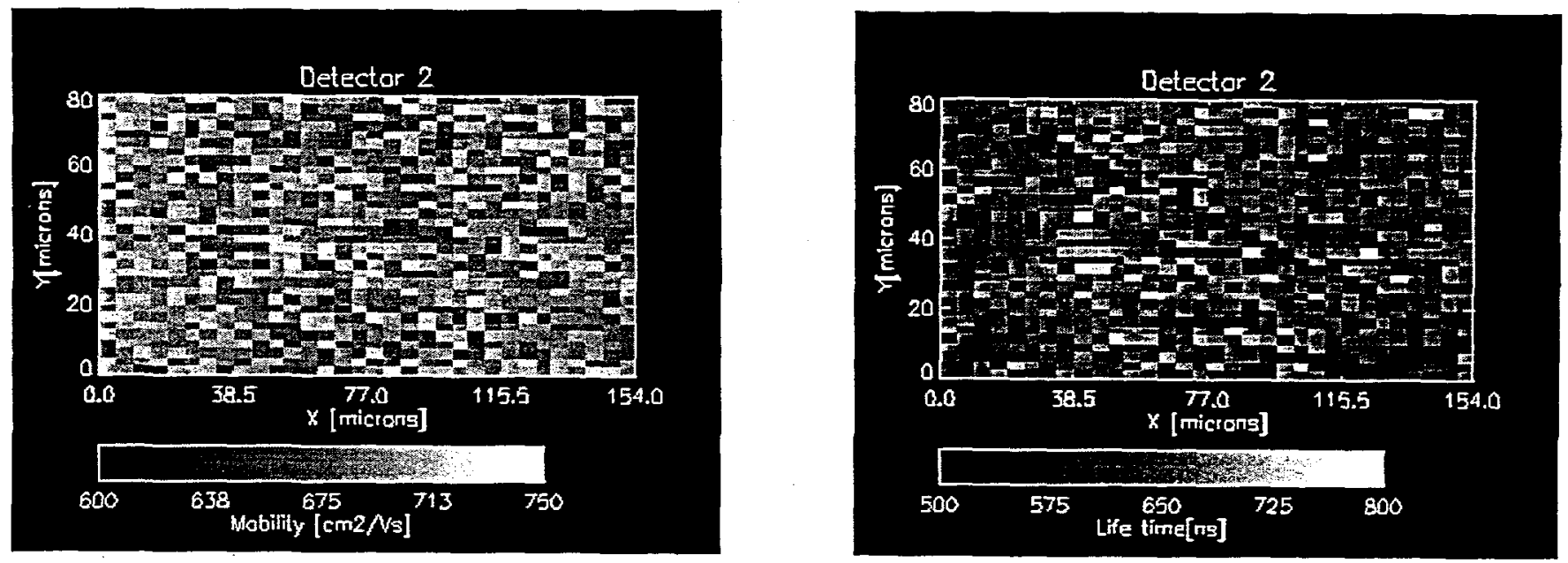

Figure $3 \mathrm{a}$ and $\mathrm{b}$ Mobility and lifetime maps for detector 2

time and the signal height were calculated. The rise time, which is what TRIBICC measures, is given by $T_{r}(E)=\frac{d}{\mu \cdot E}$.

This allows us to calculate $\mu$, the electron mobility. Using TRIBICC to measure $Q$, the $\mu \tau$ product is determined from (3.2) and the lifetime can be calculated. These values are the averages over $200 \times 180 \mu \mathrm{m}^{2}$ areas. The mobility for both detectors appears to be on the low end $\left(\mu_{1}=725 \pm 6 \mathrm{~cm}^{2} / \mathrm{Vs}\right.$ and $\left.\mu_{2}=680 \pm 7 \mathrm{~cm}^{2} / \mathrm{Vs}\right)$, while the lifetimes are closer to the literature data $\left(\tau_{1}=1.7 \pm 0.2 \mu \mathrm{s}\right.$ and $\left.\tau_{2}=0.6 \pm 0.01 \mu \mathrm{s}\right)$. Figures $3 \mathrm{a}$ and $3 \mathrm{~b}$ show the mobility and lifetime maps ( 32 pixel $\mathrm{x} 32$ pixel) over the scanned area for detector 2 . Since each point corresponds to one transient, a statistical error cannot be given for these values. At this point we should mention one drawback of using alpha particles, which are mainly used because they are easily available from radioactive sources. Also, as we mentioned above they create the charge only in the topmost layer.This can sometimes be a disadvantage, because it is very sensitive to the surface conditions of the detectors. These detectors do not always 


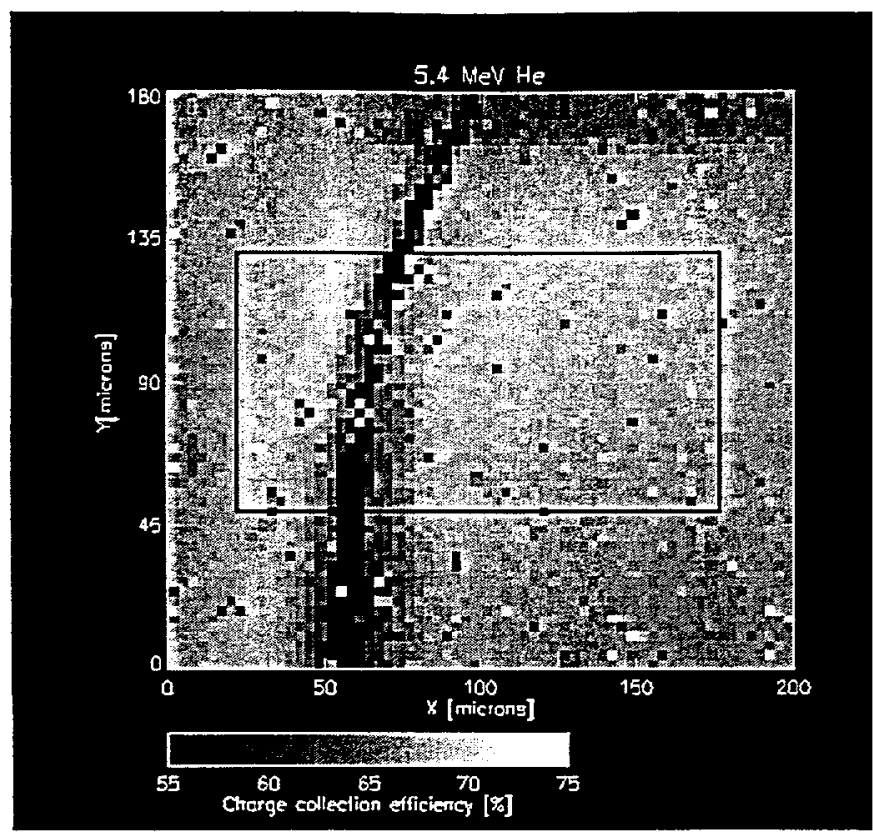

Figure 4 Charge collection efficiency map recorded using $5.4 \mathrm{MeV} \mathrm{He}$

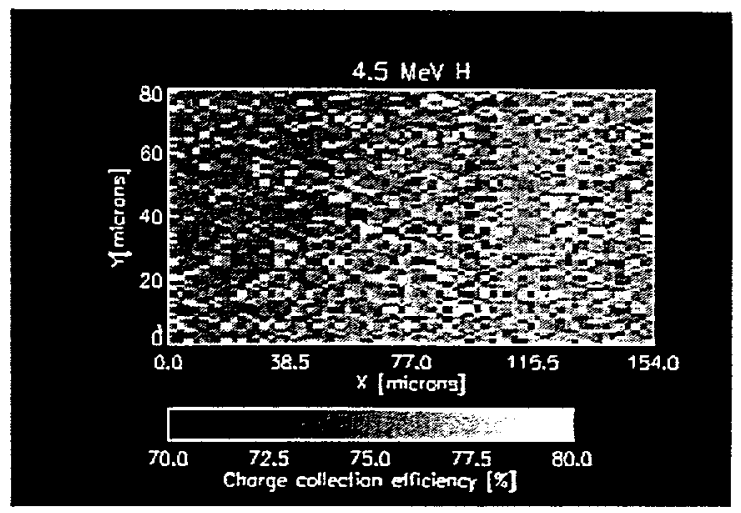

Figure 5 Charge collection efficiency of the central rectangle on Figure 4 using $4.5 \mathrm{MeV}$ protons

have perfect surfaces, many times micro-scratches and surface imperfections can be found on them. If these defects extend further than a $\mu \mathrm{m}$ they can affect the collected charge (about one tenth of the total energy is deposited in the first $\mu \mathrm{m}$ ) by trapping electrons in them. In figure 2 we also show the ionization profile of $4.5 \mathrm{MeV}$ protons. Since most of the protons' energy is deposited further below the electrode, we can expect less sensitivity to the surface conditions in case of protons. To illustrate this effect we recorded charge collection maps on a selected area of detector 2 using both $5.4 \mathrm{MeV}$ alpha particles and $4.5 \mathrm{MeV}$ protons. This particular area showed a long line across it observable by an optical microscope. The maps are shown in Figures 4 and 5. The black rectangle in Figure 4 shows the area of the proton scan. The charge collection efficiency measured with the alpha particles at the line is at least $20 \%$ lower than that in the surrounding area. On the other hand the charge collection efficiency measured by the proton beam is practically uniform over the same area (i.e. no pattern can be observed). Obviously, this imperfection extends several $\mu \mathrm{m}$ deep in the detector, which traps electrons created by the alpha particle beam. Most of the electrons created by the proton beam are not affected since they are generated well below this surface imperfection. Only the holes would be affected in this case but their contribution is negligible. The same area was measured using TRIBICC with both the alpha particles and protons. The signal height showed the same pattern for the alpha particles, no pattern for protons, and no pattern was observed in the transit time in either case.

\subsection{Lateral IBICC and TRIBICC}

Several groups are using lateral IBICC for studying CZT, CdTe, and Si detectors ${ }^{6,7,8,9}$. In this measurement the side of the detectors is scanned between the electrodes. The charge collection efficiency profile should follow (3.1) in case of constant electron and hole drift length. In principle this measurement allows us to determine both the electron and hole drift lengths carrying out the measurement at a single bias voltage. If the electric field is not constant along the detector's axis we have to take into account the change in the drift lengths between the electrodes. That leads to a more complicated expression that in most cases is hard to fit to the measurements. It is further complicated by the shaping time of the spectroscopy amplifier. If the drift time is longer than the shaping time (3.1) will not be valid anymore because the measured signal will not be proportional to $Q$. A computationally intensive correction can be made but information is lost because of the short shaping times. A recent article has a very good discussion of the theory of this method ${ }^{10}$.

The traditional lateral IBICC is quite useful if only the electrons contribute to the collected charge or the holes have negligible contribution only. Still the use of very short shaping times should be avoided. The effect of the shaping time on the lateral IBICC profile is shown in Figure 6. These profiles were measured on detector 1 at $200 \mathrm{~V}$ bias. A $180 \mu \mathrm{m}$ wide stripe was scanned between the electrodes and the charge collection efficiency was averaged over $3 \mu \mathrm{m} \times 180 \mu \mathrm{m}$ areas. The expected drift time for electrons from the measured mobility is about $270 \mathrm{~ns}$. We can expect that the IBICC profile saturates for 


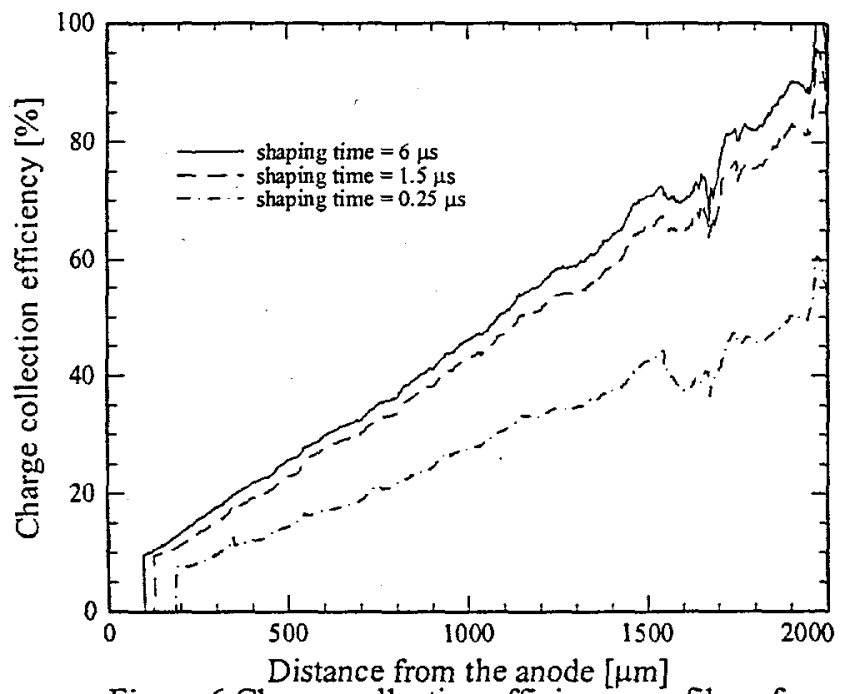

Figure 6 Charge collection efficiency profiles of detector 1 at $200 \mathrm{~V}$ bias with different shaping shaping times over a $\mu$ s. It is no surprise that the IBICC profile at $0.25 \mu$ s shaping time is significantly lower than at larger shaping times. Also we can notice that while the shapes of the curves at 6 $\mu$ s and $1.5 \mu \mathrm{s}$ are basically identical, the shape of the $0.25 \mu \mathrm{s}$ profile is significantly different. The signal increases more slowly as we approach the cathode, the short shaping time has more effect when the electrons have to travel longer distances. Since the profiles decrease almost linearly toward the anode and we can extrapolate it to almost zero at the anode we can conclude that there is little contribution from the holes. The dip around $1500 \mu \mathrm{m}$ is due to some imperfection of the surface. A full map of the IBICC stripe is shown in Figure 7. A line around $1500 \mu \mathrm{m}$ is clearly seen across the whole stripe, otherwise it seems homogenous.

A better, although much slower way to measure the charge collection efficiency profile is to do TRIBICC. This way the shaping time does not effect the signal height. Figure 8 shows the TRIBICC profile of the same sample averaged over a $16 \mu \mathrm{m}$ wide stripe around the middle line of the IBICC scan. This profile is almost

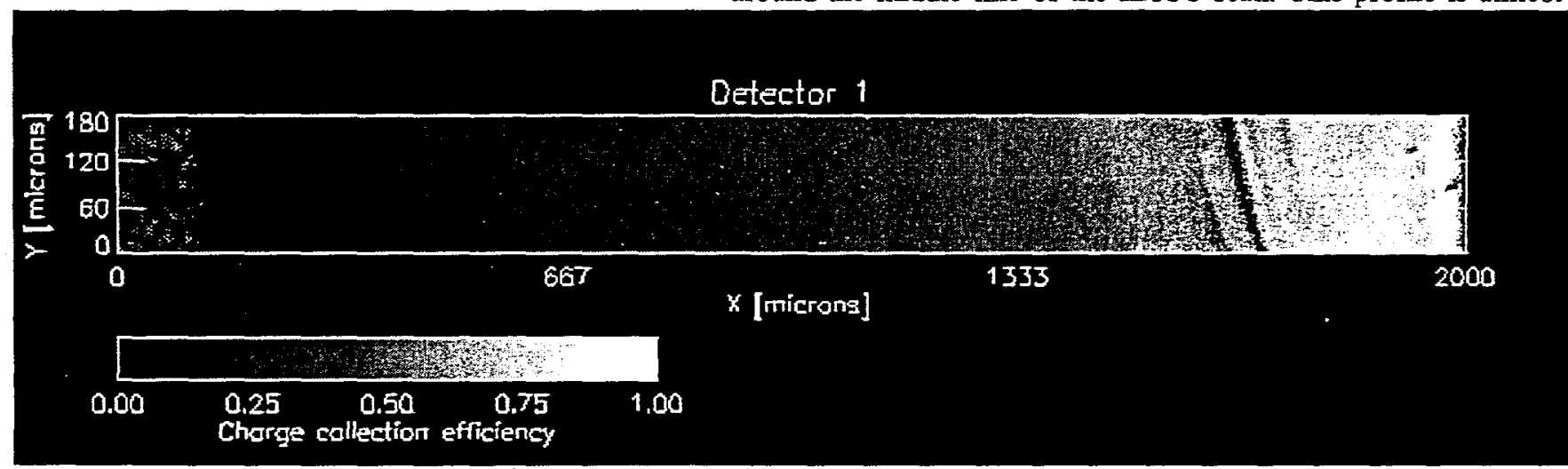

Figure 7 Lateral IBICC map of detector 1 at $200 \mathrm{~V}$ bias

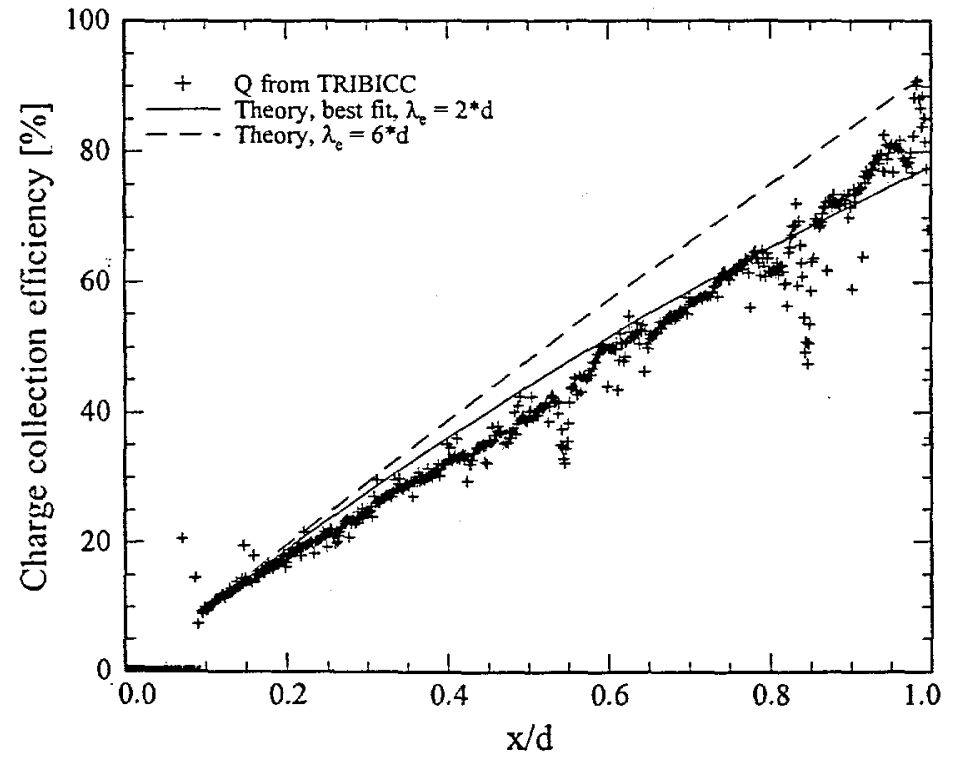

Figure 8 TRIBICC profile of detector 1 at $200 \mathrm{~V}$ bias exactly the same as the IBICC profile except the dip around $1500 \mu \mathrm{m}$ seems sharper and there is a second dip around $1100 \mu \mathrm{m}$. Both of these can be explained if we have a look at Figure 7 . The line at $1500 \mu \mathrm{m}$ does not run perpendicular to the scan but rather diagonally. Averaging over the whole $180 \mu \mathrm{m}$ area makes the dip wider and shallower. A dark spot around $1100 \mu \mathrm{m}$ in the centerline of the stripe explains the second dip, that is some small imperfection and unfortunately the TRIBICC scan ran through that spot. This profile, which is almost linear, confirms the lack of holes. The two lines show the theoretical profiles, the solid line is the profile calculated using the previously determined $\mu \tau$ product. It matches up very well at the cathode but then overestimates it until it reaches about $200 \mu \mathrm{m}$ from the anode. The dashed line is the best fit to the experimental data using about one third of the previously calculated $\mu \tau$ value. This TRIBICC profile exhibits the same sharp peak at the cathode as all the IBICC scans. This explains why the frontal IBICC and TRIBICC gave a 


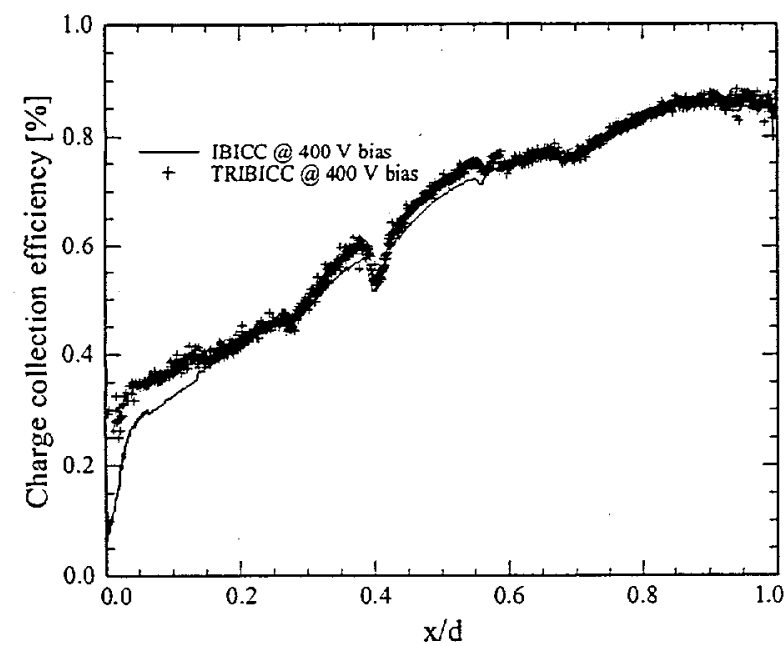

Figure 9 IBICC and TRIBIC profiles of detector 2 at $400 \mathrm{~V}$ bias with $4.5 \mathrm{MeV}$ protons

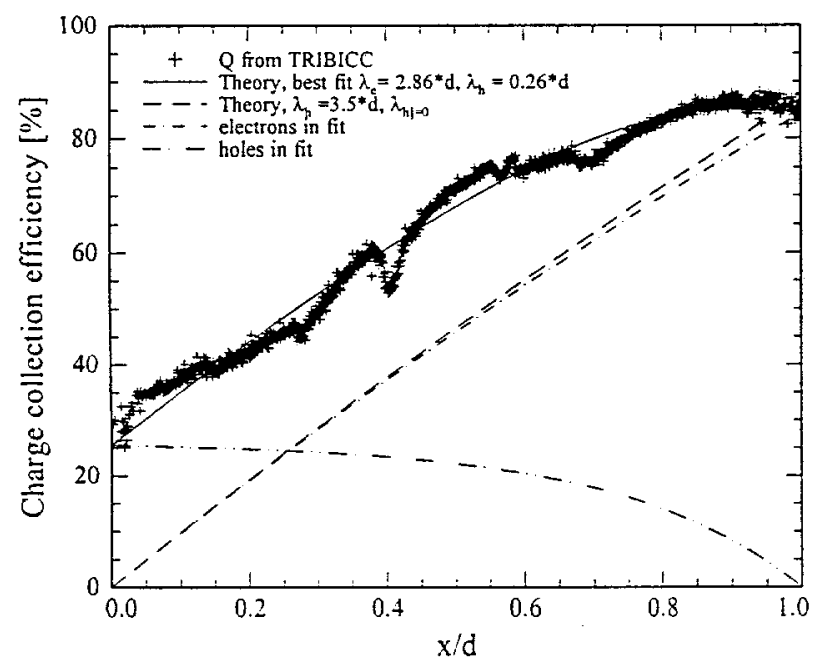

Figure 10 TRIBICC measurement and theory for detector 2 at $400 \mathrm{~V}$ bias

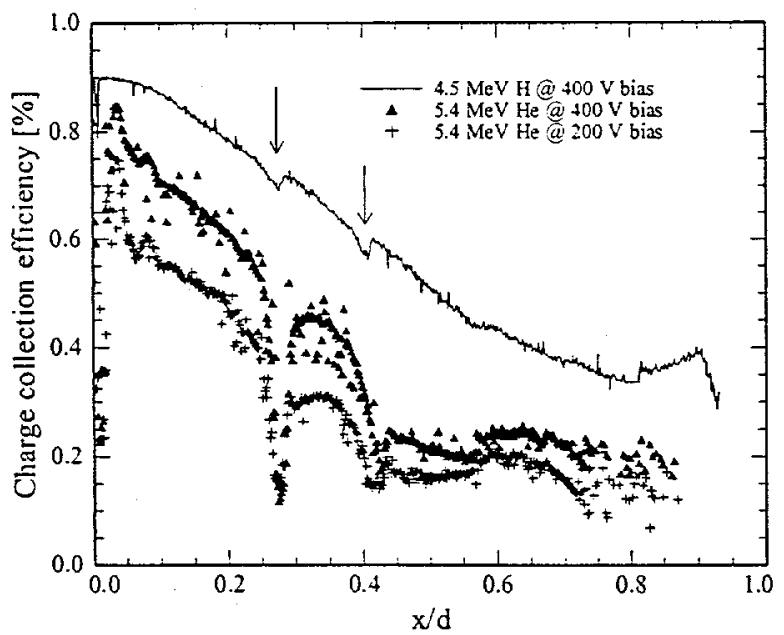

Figure 11 Proton and helium TRIBICC profiles on detector 2 higher $\mu \tau$ value, since they measured exactly that peak. The deviation from the fit and the spike at the cathode indicate a non-uniform electric field, a higher than average field at the cathode that was observed by several groups. We have not observed that large spike at the cathode on the previous samples, its origin must be related to the way the contacts were made (sputtering of platinum). The spike shows up the same way on the other side of the detector if the bias is reversed.

An example when the TRIBICC measurement can really provide more information is the experiment on detector 2 . Figure 9 shows the IBICC and TRIBICC profiles of detector 2 at $400 \mathrm{~V}$ bias measured by 4.5 $\mathrm{MeV}$ protons. The IBICC profile itself already indicates that in this case we have some contribution from the holes. The TRIBICC profile is very similar to the IBICC profile until about $400 \mu \mathrm{m}$ to the anode, then it is significantly higher indicating that the spectroscopy amplifier's shaping time was cutting out the contribution of the slower holes. The $30 \%$ charge collection efficiency at the anode shows significant hole contributions. The drift time (the rise time of the signal)

around the anode was about $1-2 \mu$ s which gives about $70 \mathrm{~cm}^{2} / V s$ mobility for the holes. In this case we did not find a spike at the surface which indicates that the electric field might be smoother than in detector 1. Figure 10 shows the same TRIBICC profile with a calculation using the $\mu \tau$ from the frontal TRIBICC measurement and no holes (dashed line). The solid line is the best fit assuming both electrons and holes. The $\mu \tau$ values for the electrons and holes from the fit are $\mu \tau_{\mathrm{e}}=2.86 \times 10^{-4} \mathrm{~cm}^{2} / \mathrm{V}$ and $(\mu \tau)_{\mathrm{h}}=2.6 \times 10^{-5} \mathrm{~cm}^{2} / \mathrm{V}$ respectively. This gives $0.37 \mu \mathrm{s}$ for the hole lifetime. The electron $\mu \tau$ value is very close to that calculated from the frontal TRIBICC measurements $\left(3.56 \times 10^{-}\right.$ $\left.{ }^{4}\right)$.

At this point we have to return to the problem of what kind of beam is the best for these experiments. Since this detector was already passivated we did not want to repolish the side. First we tried to use alpha particles then we switched to protons. The comparison of the alpha and proton TRIBICC profiles is shown in Figure 11. Since the surface was not very well polished the charge collection efficiency is smaller for the alpha particles and it even drops further as the alpha particles go through some surface defects. On the other hand the proton profile is quite smooth, but the dips are still present as indicated by the arrows. Although, the surface defect/damage reaches deeply into the detector its effect on the proton created electron-hole pairs is small.

\subsection{Damage}

Unfortunately, this method is not completely non-destructive. Part of the ion's energy is used to create vacancies that become traps for the charge carriers. Studies ${ }^{11}$ in the past found that significant performance degradation occurs above $10^{10}$ particles $/ \mathrm{cm}^{2}$. In our experiments we tried to keep the irradiation dose low, well below $10^{9}$ particles $/ \mathrm{cm}^{2}$. Even at this low dose we found that the peak of a homogenous spot shifted about $2 \%$ for both detectors above $10^{9}$ particles $/ \mathrm{cm}^{2}$. This does not seem to be of a problem but it is definitely observable. Figure 12 shows a scan over half of an irradiated spot and half of a virgin spot after $2 \times 10^{9}$ particles $/ \mathrm{cm}^{2}$. The damaged region can be clearly seen on the left. Although the peak height shifted down only by $2 \%$ the 


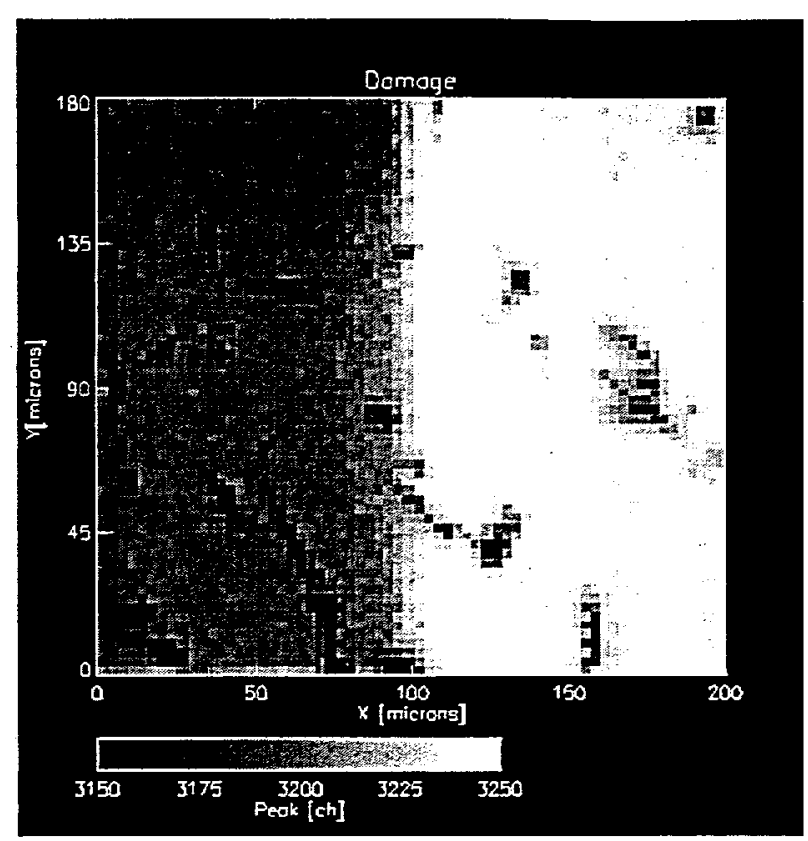

Figure 12 IBICC scan over half of an irradiated spot of detector 1

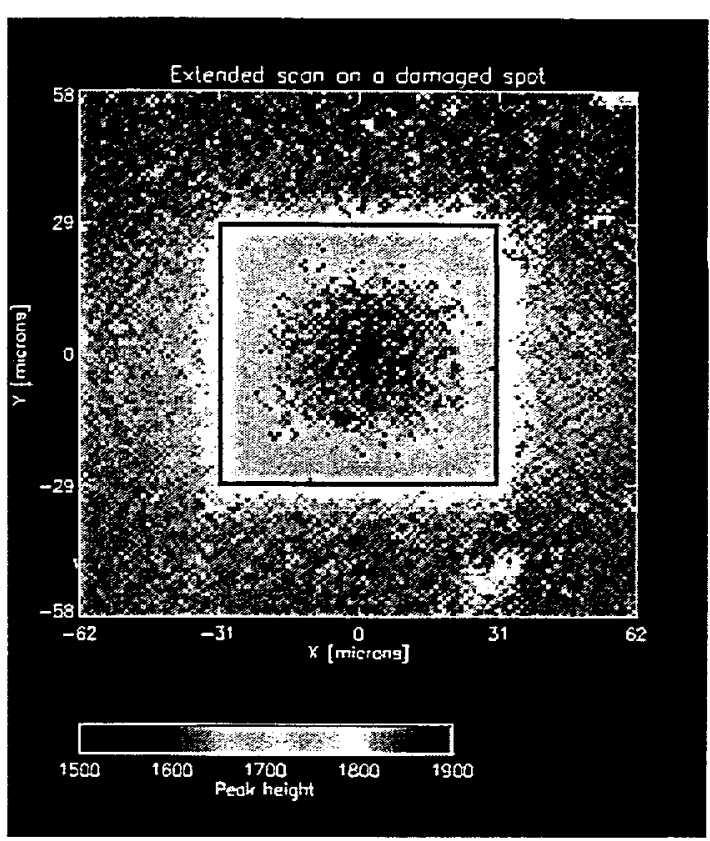

Figure 13 Extended scan on a damaged spot after $5 \times 10^{10}$ particle $/ \mathrm{cm}^{2}$ dose

FWHM of the peak changed significantly. The reason for this large change is that the charge collection efficiency does not change uniformly over the irradiated area. The degradation is faster in the center and slower on the edges and in the corners. After high dose irradiation a characteristic pattern can be observed, there is a more or less uniform, low charge collection efficiency in the center region and it gradually increases toward the edges. This border area is several $\mu \mathrm{m}$ wide, and cannot be explained by the overlapping cascades or scans. Figure 13 shows an extended scan over a previously irradiated spot. The total dose was $5 \times 10^{10}$ particles $/ \mathrm{cm}^{2}$. The border region is more than $10 \mu \mathrm{m}$ wide. The brief qualitative explanation is as follows. The alpha particles create most of the vacancies at the end of their range so after a large dose there is a highly damaged thin layer $(\sim 1 \mu \mathrm{m})$ around $20 \mu \mathrm{m}$ below the cathode. Since practically all the electron-hole pairs are created between the cathode and this highly damaged (full of traps) region, all the electrons have to go through the damaged region. While the electrons are drifting from the cathode toward the anode they are diffusing in the plane perpendicular to the direction of the drift. The damaged region is not only localized in depth but it is about as large as the scanned spot. Therefore, electrons starting closer to the edge have a chance to get outside of the scanned spot by the time they reach the damaged region. This way they do not go through the damaged layer and do not get trapped in it. The detailed model ${ }^{12}$ and calculations show that the width of the border layer is only a function of the electric field in the region between the cathode and the damaged layer. In order to obtain this wide border region this electric field must be significantly lower than the nominal field. This assumption is supported by the fact that the signals from the center of the damaged spot have longer rise times than the ones from the edges. The origin of the low electric field is assumed to be trapped negative charge in the damaged layer. In order to avoid damage the maximum dose should be kept well below $10^{9}$ particles $/ \mathrm{cm}^{2}$ (or 10 particles $/ \mu \mathrm{m}^{2}$ ).

\section{SUMMARY}

The IBICC and TRIBICC techniques that are used routinely to study microelectronic devices are perfectly suitable to investigate the electronic transport properties of gamma-ray detectors. Frontal IBICC and TRIBICC allow the calculation of electron mobility and lifetime with $\mu \mathrm{m}$ spatial resolution, although it can give false result if the electric field in the detector is far from uniform. The lateral version of the above techniques provides information about the electric field distribution along the detector's axis and makes it possible to calculate the hole drift length. When the hole contribution is significant, TRIBICC should be used rather than IBICC to avoid losing information about the hole collection efficiency because of the short amplifier shaping times.

We found that the electric field is more uniform in the passivated, doped detector than in the other one. The detector with the sputtered platinum contacts showed a large electric field at the cathode and smaller field in the bulk of the detector. This detector had better $\mu \tau$ for the electrons but no hole transport could be observed. In the passivated detector we found significant hole contribution. 
Since $\mathrm{MeV}$ alpha particles have short range in these detectors they are sensitive to the surface conditions of the detectors which can be a disadvantage. In these cases the use of a proton beam is preferred because it creates the charge in larger depth; therefore, the results are not affected by the surface conditions. Also, a proton beam causes less damage to the detector crystal itself.

Although, these techniques are virtually non-destructive techniques the applied dose should be watched carefully. Even $10^{9}$ particles $/ \mathrm{cm}^{2}$ can cause charge collection efficiency and resolution degradation. The damage to the detectors will eventually limit the ultimate lateral resolution to about $0.5 \mu \mathrm{m}$ for alpha particles and to $0.2 \mu \mathrm{m}$ for protons.

\section{ACKNOWLEDGEMENT}

The authors would like to acknowledge Dr. Csaba Szeles of eV Products for providing Detector 1 and Dr. Vitaly Komar of the Institute for single Crystals for providing Detector 2 for this study.

\section{REFERENCES}

\footnotetext{
${ }^{1}$ B.A. Brunett, J.M. van Scyoc, H. Yoon, T.S. Gilbert, T.E. Schlesinger, J.C .Lund, and R.B. James, "Mapping of large area Cadmium Zinc Telluride (CZT) wafers: Apparatus and Methods", Mat. Res. Soc. Symp. Proc., 487. 39-44, 1998.

${ }^{2}$ B.L. Doyle and N. Wing, "3-dimensional profiling with the Sandia muclear microprobe", IEEE Transactions on Nuclear Science, $\underline{30}, 1214-1219,1983$

${ }^{3}$ H. Schöne, D.S. Walsh, F.W. Sexton, B.L. Doyle, P.E. Dodd, J.F. Aurand, N. Wing, N., "Time-resolved ion beam induced charge collection (TRIBICC) in micro-electronics", Nucl. Instr. and Meth. B158, 424-431, 1999.

${ }^{4}$ K. Hecht, Z. Physik, 77, 235, (932

${ }^{5}$ G.F. Knoll and D.S. McGregor, "Fundamentals of semiconductor detectors for ionizing radiation", Mat. Res. Soc. Symp.

Proc., 302, 3-17, 1993.

${ }^{6}$ C. Manfredotti, F. Fizotti, P. Posello, and E. Vittone, Nucl. Instr. Meth., "A realistic simulation of gamma-ray detectors", A380, 145-147, 1996.

${ }^{7}$ C. Manfredotti, F. Fizotti, P. Polesello, P.P. Trapani, E. Vittone, M. Jaksic, S. Fazinic, and I. Bogdanovic, "Investigation on the electric field profile in CdTe by ion beam induced current", Nucl. Instr. Meth., A380, 136-140, 1996.

${ }^{8}$ Z. Pastuoviz, M. Jaksic, R.B. James, K. Chattopadhyay, X. Ma, and A. Burger, "Influence of electrical contacts on charge collection profiles in CdZnTe studied by IBICC", in Proc. of $11^{\text {th }}$ International Workshop on Room Temperature Semiconductor X- and Gamma-ray Detectors and Associated Electronics, October 11-15, 1999, Vienna, Austria

${ }^{9}$ G. Vizkelethy, B.A. Brunett, D.S. Walsh, R.B. James, and B.L. Doyle, "Investigation of the electronic properties of Cadmium Zink Telluride (CZT) detectors using a nuclear microprobe", in Proc. of $11^{\text {th }}$ Intemational Workshop on Room Temperature Semiconductor X- and Gamma-ray Detectors and Associated Electronics, October 11-15, 1999, Vienna, Austria ${ }^{10}$ E. Vittone, F. Fizzotti, A. Lo Giudice, C. Paolini, and C. Manfredotti,"Theory of ion beam induced charge collection in detectors based on the extended Shockley-Ramo theorem", Nucl. Instr. Meth., B161-163, 446-451, 2000.

${ }^{11}$ L.A. Franks, B.A. Brunett, R.W. Olson, D.S. Walsh, G. Vizkelethy, L.I. Trombka, B.L. Doyle, and R.B. James, "Radiation damage measurements in room-temperature semiconductor radiation detectors ", Nucl. Instr. Meth., A428, 95-101, 1999.

${ }^{12}$ B.L. Doyle, G. Vizkelethy, and D.S. Walsh, "Ion beam induced charge collection (IBICC) studies of cadmium zinc telluride (CZT) radiation detectors", Nucl. Instr. Meth., B161-163, 457-461, 2000.
} 